\title{
Artificial photosynthesis
}

\author{
Devens Gust*, Thomas A. Moore, Ana L. Moore
}

Center for Bio-Inspired Solar Fuel Production, Arizona State University, Tempe, AZ 85287, USA.

*Corresponding author: gust@asu.edu

Received: 27 August 2013; Accepted: 4 September 2013

Photosynthesis is the largest-scale, longest-tested, and most important solar energy conversion system on earth. Photosynthetic organisms provide fuel for most of the biosphere, and generated the fossil fuels that account for about $85 \%$ of the energy used today by human technology. In a time in which humans are searching for ways to limit society's contribution to climate change and reduce fossil fuel dependence and its associated geopolitical and supply problems, it is natural to turn to photosynthesis for solutions. Researchers are searching for ways to improve the efficiency ( $<1 \%$ in most cases) and environmental impacts of using natural organisms to supply fuels. However, there is another approach: artificial photosynthesis. The idea of using the basic science underlying photosynthesis in the design of systems for making solar fuels has been discussed for over 100 years (Ciamician 1912). In spite of this, no viable technology for synthesizing fuels using sunlight can currently compete with fossil fuel utilization. A great deal of progress has been made in recent years, however. Some of this progress will be illustrated here with examples from our laboratories and those of our colleagues.

\section{ARTIFICIAL PHOTOSYNTHETIC REACTION CENTERS}

In photosynthetic organisms, reaction center proteins convert light excitation energy into chemical potential energy that can be used to power metabolism. These reaction centers use the phenomenon of photoinduced electron transfer to carry out this conversion. Light energy is used to move an electron from donor chlorophyll to an electron acceptor, creating a charge-separated state. Subsequent electron transfers move the resulting oxidizing and reducing equivalents away from the site of charge separation to catalytic sites where, in oxygenic photosynthesis, they are used to oxidize water to oxygen and carry out reductive chemistry that can form fuels, respectively.

Photoinduced electron transfer is also characteristic of artificial reaction centers. An example is shown in Figure 1.

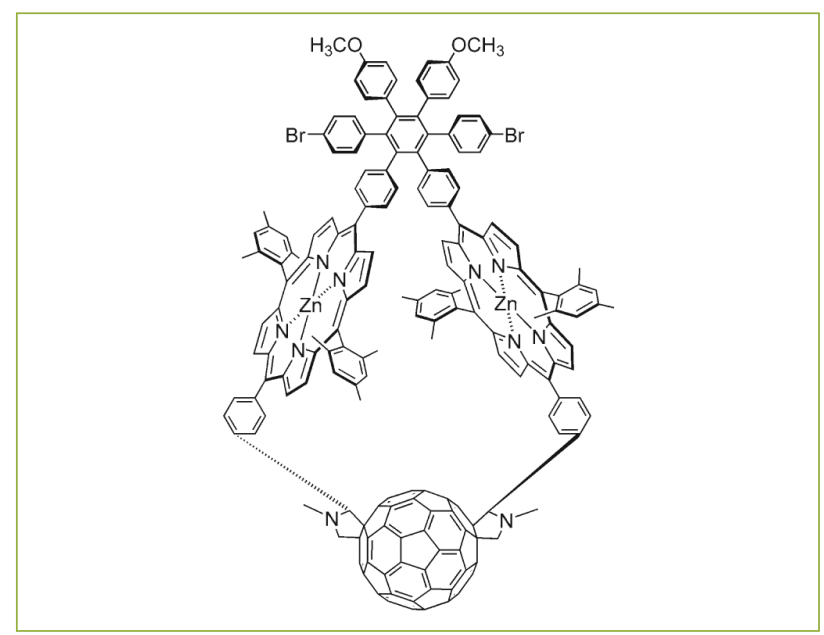

Figure 1. Artificial photosynthetic reaction center 1. 
In this synthetic molecule 1 (Garg et al.2011), two porphyrins (similar in structure and properties to chlorophylls) are held in an arrangement reminiscent of the chlorophyll "special pairs" in bacterial photosynthesis, and covalently bound to a fullerene moiety. Transient spectroscopic studies show that after a porphyrin absorbs light, its excited singlet state transfers an electron to the fullerene with a time constant of 1.1 ps to give the $\mathrm{P}^{++}-\mathrm{C}_{60}{ }^{-\cdots}$ charge separated state with a quantum yield of $100 \%$.

Molecule 1 satisfies some of the requirements of a good artificial photosynthetic reaction center in that it absorbs visible light and converts the energy of essentially every absorbed photon into electrochemical energy. However, the charge separated state that is formed only lasts $2.7 \mathrm{~ns}$ before it returns to the ground state, liberating the stored energy as heat. Doing useful chemistry with the charge-separated state would be easier if it had a longer lifetime.

Artificial reaction center 2 (Figure 2) solves this problem by borrowing a concept from natural photosynthesis (Kodis et al. 2002). In natural reaction centers, the initial photoinduced electron transfer reaction is followed rapidly by secondary electron transfer steps in which the charges are shifted to additional donor and acceptor moieties in order to decrease the electronic interaction between them, and thereby decrease the rate of charge recombination. In molecule 2 , the excited free base porphyrin shown in red donates an electron to the attached fullerene with a time constant of 25 ps in 2-methyltetrahydrofuran solvent. The quantum yield is $98 \%$. Prior to recombination of the resulting charge separated state $(\tau=3.0 \mathrm{~ns})$, the positive charge migrates to the zinc porphyrin array with a time constant of $380 \mathrm{ps}$. The greater

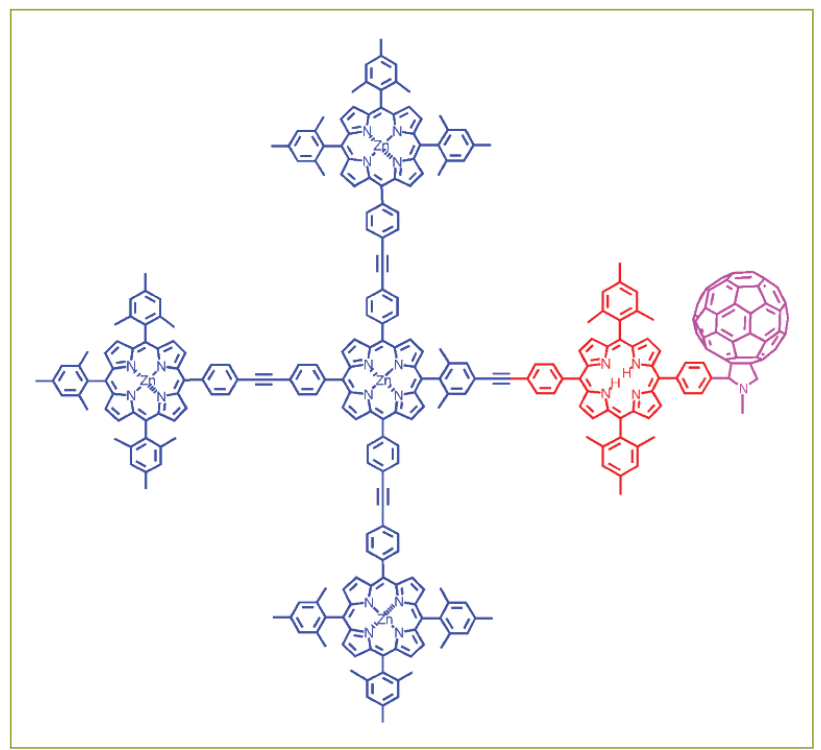

Figure 2. Artificial antenna-reaction center 2. separation of charge resulting from this second electron transfer reaction increases the lifetime of the charge separated state to 240 ns. The added complexity results in an almost 100-fold increase in the lifetime of the charge-separated state, which makes it much easier to imagine using these energetic species to do useful chemistry.

\section{ARTIFICIAL ANTENNA SYSTEMS}

Most of the sunlight used for photosynthesis is absorbed by antenna chromophores, and not by the reaction centers. A wide variety of often complex antenna systems are found in photosynthetic organisms. Similarly, a variety of artificial photosynthetic antennas have been studied, although none of them approach the size and complexity of the natural antennas. A successful antenna (either natural or artificial) must both absorb light efficiently and transport its energy efficiently to a reaction center. Molecule 2 illustrates the idea of an artificial antenna-reaction center complex. In $\mathbf{2}$, the four zinc-containing porphyrins absorb light in spectral regions different from those where the red free base porphyrin absorbs. When one of the peripheral zinc porphyrin moieties absorbs light, it transfers the excitation energy by a singlet energy transfer mechanism to the central zinc porphyrin with a time constant of $50 \mathrm{ps}$. The central zinc porphyrin can both exchange energy with the other zinc porphyrins and transfer excitation to the free base porphyrin, whose first excited singlet state lies at lower energy. This energy transfer to the free base has a time constant of 30 ps. The result of these various energy transfer reactions is that excitation of any of the zinc porphyrins is transferred to the free base porphyrin with ca. $98 \%$ efficiency. Once at the free base porphyrin, it can be used for electron transfer as discussed above. We have reported a variety of artificial antenna systems that use different chromophores to harvest efficiently various regions of the solar spectrum (Terazono et al. 2005, Kodis et al. 2006, Terazono et al. 2009, Gust et al. 1989, Gust et al. 2001, Gust et al. 2009).

\section{FUEL PRODUCTION}

To be useful, the electrochemical energy stored in the charge separated state of a photosynthetic reaction center must be used to make a fuel or electricity. In natural photosynthesis, this energyis used to make ATP and NAD $(P) H$, which are employed in carbon fixation or synthesis of hydrogen. Making a fuel using artificial photosynthesis has proven challenging. Figure 3 shows a step towards this goal. It is a photoelectrochemical cell that uses sunlight to split water to oxygen and hydrogen gas (Youngblood et al. 2009). The system consists of a buffered aqueous solution that contains two electrodes. The photoanode (on the left in Figure 3) is 
formed on conductive glass coated with nanoparticulate titanium dioxide. A ruthenium-based dye is adsorbed onto the surface of the oxide, and bears in turn an iridium oxide nanoparticle. Excitation of the dye is followed by electron injection into the $\mathrm{TiO}_{2}$ electron acceptor, much as occurs in the other artificial reaction centers discussed above. The injected electrons move through the nanoparticles and into the conductive electrode, and then via a wire to a platinum cathode, where they reduce hydrogen ions to hydrogen gas. The oxidized dye at the photoanode is reduced by electron donation from the iridium oxide nanoparticles. Accumulation of sufficient oxidizing equivalents on a nanoparticle is followed by oxidation of water to oxygen gas, hydrogen ions, and electrons which regenerate the catalyst.

Thus, the overall function of the cell in Figure 3 is the "splitting" of water to oxygen gas and hydrogen fuel using

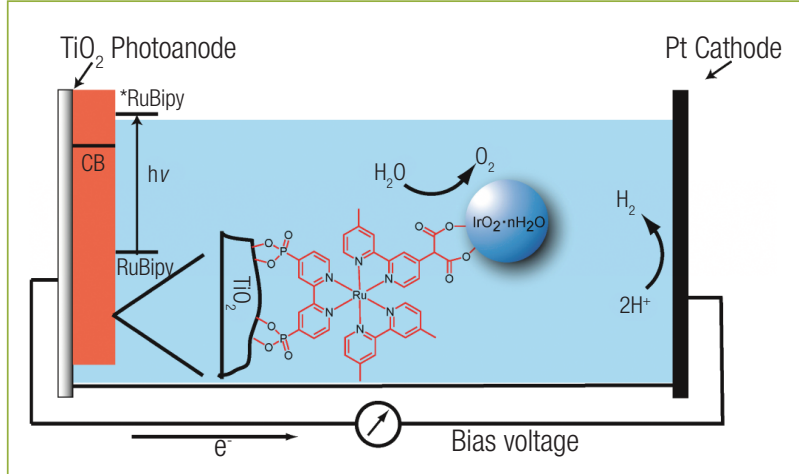

Figure 3. Photoelectrochemical cell for water splitting (Youngblood et al. 2009).

\section{REFERENCES}

Ciamician G (1912) The photochemistry of the future. Science 36:385-394.

Garg V, Kodis G, Chachisvilis M, Hambourger M, Moore AL, Moore TA, Gust D (2011) Conformationally constrained macrocyclic diporphyrin-fullerene artificial photosynthetic reaction center. Journal of the American Chemical Society 133:2944-2954.

Gust D, Moore TA (1989) Mimicking photosynthesis. Science 244:35-41.

Gust D, Moore TA, Moore AL (2001) Mimicking photosynthetic solar energy transduction. Accounts of Chemical Research 34:40-48.

Gust D, Moore TA, Moore AL (2009) Solar fuels via artificial photosynthesis. Accounts of Chemical Research 42:1890-1898. sunlight. Although this cell illustrates the principle of fuel production using sunlight, it is very inefficient (quantum yield of $\mathrm{O}_{2}$ production $=0.9 \%$ ), and requires an electrical bias voltage to function. Additional development is expected to increase this efficiency. For example, the use of a chemical electron relay between the oxidized dye and the nanoparticle has increased the efficiency $300 \%$ (Zhao et al. 2012). This relay is based on the tyrosine-histidine electron relay that performs a similar function in photosystem II of photosynthetic organisms.

Future developments in the solar fuels area are expected to include catalysts that do not depend on rare and expensive metals such as iridium, platinum or ruthenium. Biology has developed useful catalysts for water oxidation (PSII) and hydrogen production (hydrogenases) that rely on abundant metals such as manganese, calcium, iron and nickel, and these enzymes may point the way to the design of useful synthetic analogs.

\section{CONCLUSIONS}

In this brief report, we have illustrated the realization of artificial photosynthetic reaction centers, antennas, water oxidation systems, and hydrogen production systems. All of these owe their basic design parameters to processes found in natural photosynthetic organisms. Other aspects of energy utilization by living organisms, such as photoprotection and photoregulation of photosynthesis, conversion of carbon dioxide to useful fuels and nitrogen fixation, are inspiring artificial analogs as well. Biology has spent billions of years developing strategies for using environmental resources to promote life while preserving an overall environment conducive to life. Perhaps we can learn to do the same.

Kodis G, Liddell PA, de la Garza L, Clausen PC, Lindsey JS, Moore AL, Moore TA, Gust D (2002) Efficient energy transfer and electron transfer in an artificial photosynthetic antennareaction center complex. The Journal of Physical Chemistry $A$ 106:2036-2048.

Kodis G, Terazono Y, Liddell PA, Andréasson J, Garg V, Hambourger M, Moore TA, Moore A L, Gust D (2006) Energy and photoinduced electron transfer in a wheel-shaped artificial photosynthetic antenna-reaction center complex. Journal of the American Chemical Society 128:1818-1827.

Terazono Y, Liddell PA, Garg V, Kodis G, Brune A, Hambourger M, Moore TA, Moore AL, Gust D (2005) Artificial photosynthetic antenna-reaction center complexes based on a hexaphenylbenzene core. Journal of Porphyrins and Phthalocyanines 9:706-723. 
Terazono Y, Kodis G, Liddell PA, Garg V, Moore TA, Moore AL, Gust D (2009) Multiantenna artificial photosynthetic reaction center complex. The Journal of Physical Chemistry 113:7147-7155.

Youngblood WJ, Lee SHA, Kobayashi Y, Hernandez-Pagan EA, Hoertz PG, Moore TA, Moore AL, Gust D, Mallouk TE (2009) Photoassisted overall water splitting in a visible light-absorbing dye-sensitized photoelectrochemical cell. Journal of the American Chemical Society 131:926-927.
Zhao Y, Swierk JR, Megiatto JD, Sherman BD, Youngblood WJ, Qin D, Lentz DM, Moore AL, Moore TA, Mallouk TE (2012) Improving the efficiency of water splitting in dye-sensitized solar cells by using a biomimetic electron transfer mediator. Proceedings of the National Academy of Sciences USA 109:15612-15616. 